\title{
New Representations for the Madelung Constant
}

\author{
Richard E. Crandall
}

\section{CONTENTS}

1. Overview of the Madelung Problem

2. Analytic Consequences of the Andrews Identity

3. Connection with Multiple Zeta Sums

4. An $\eta$-Series for $M(s)$

5. Integral Representations for the Madelung Constant

6. Number-Theoretical Implications: Sums of Three Squares

7. Open Problems

Acknowledgments

References
From a modern theta-function identity of G. E. Andrews we derive new representations for the celebrated Madelung constant and various of its analytic relatives. The method leads to connections with the modern theory of multiple zeta sums, generates an apparently entire " $\eta$ series" representation, and, for the Madelung constant in particular, yields a finite-integral representation. These analyses suggest variants of the Andrews identity, leading in turn to number-theoretical results concerning sums of three squares.

\section{OVERVIEW OF THE MADELUNG PROBLEM}

The Madelung constant - we shall call it $M$ - of chemistry and physics enjoys an illustrious yet notorious history. Since $M$ is essentially the binding energy density of an ideal (sodium-chloride) crystal structure, the mathematical problem of assigning a theoretical value for $M$ has direct implications for the chemical laboratory. It can be argued that a branch of mathematics - the analysis of lattice sums and multidimensional zeta functions - grew in large measure out of fascination with the Madelung problem. A very brief history of these developments runs as follows, where we have drawn on previous overviews, such as the monumental review work of [Glasser and Zucker 1980] and the interdisciplinary approach of [Buhler and Wagon 1996].

The science of lattice sums was begun by Appell in the late 19th century, in the context of periodic solutions to certain differential equations of physics. His efforts led to expansions for energy density, which analysis seems to have been overlooked over many subsequent years. In 1918 Madelung attempted a theoretical summation over parallel planes of charge, in this way providing the first reasonable numerical value $M \sim-1.7435 \ldots$, which rings within 0.2 per cent of true. In 1921 Ewald worked out a convergent series expansion applicable to arbitrary crystals, about which expansion we have more to say

(C) A K Peters, Ltd. 1058-6458/1999 \$0.50 per page Experimental Mathematics 8:4, page 367 
below. For the rest of the 20th century, new computational representations have arisen, usually in the form of convergent series. However - and here is the notorious aspect to which we have alluded while motivation for both numerical and theoretical evaluations of $M$ has persisted throughout the 20th century, and though we now possess a numerical value to thousands of decimal digits, the Madelung constant has never been given a theoretical evaluation in any convenient, closed form. Throughout the present treatment, we take a number to have been "evaluated" - this is a qualitative term - if it is represented as a suitable combination of fundamental numbers. In particular, specific values of any $L$-series such as the one-dimensional Riemann zeta function may appear, as may explicit algebraic numbers such as $\sqrt{2}$, values such as $\Gamma\left(\frac{1}{4}\right)$ of standard special functions, and so on.

We take $M$ to be the analytic continuation, to $s=\frac{1}{2}$, of a specific, three-dimensional Epstein zeta function:

$$
M(s)=\sum_{x, y, z \in Z}^{\prime} \frac{(-1)^{x+y+z}}{\left(x^{2}+y^{2}+z^{2}\right)^{s}},
$$

where $\sum^{\prime}$ means that the origin singularity is to be avoided. The number $M=M\left(\frac{1}{2}\right)$ is the celebrated Madelung constant. More precisely, $M$ is the potential energy of the origin charge in a (sodiumchloride-type) lattice of charges, calculated as the sum of potentials $\pm 1 / r$ for distances $r$ to various lattice sites. We point out that other periodic crystal structures give rise to similar constants, again as analytic continuation evaluations of a relevant Epstein zeta function, and in such cases one may speak of the "Madelung constant" for a particular crystal structure. Hereafter we focus on the (sodium chloride) $M(s)$ function.

Though the sum (1.1) for $M(s)$ converges absolutely for $\operatorname{Re} s>\frac{3}{2}$, convergence for the Madelung case $s=\frac{1}{2}$ is problematic, which is why analytic continuation must be invoked for physically meaningful instances. The literal sum, for $s=\frac{1}{2}$, definitely does not converge over ever-expanding spheres, although it does converge over ever-expanding cubes [Borwein et al. 1985; Borwein and Borwein 1994; Borwein et al. 1998a]. There is also a now-proven "Delord conjecture", saying that the energy contributions from a sphere of radius $R$ can be corrected in a delicate way - in fact in the same way that nature would neutralize an isolated sphere of lattice charges - to force convergence as $R \rightarrow \infty$ [Buhler and Crandall 1990; Buhler and Wagon 1996]. In some settings, such cancellation ideas lead to connections between crystal energies and the celebrated Euler and related constants [Kukhtin and Shramko 1993].

Although a closed form for $M$ remains elusive, various connections have been made between $M$ and similar entities, such as other Epstein zeta values. There is much literature on the theory of lattice sums, in which one can witness closed forms for analogues of the Madelung constant in dimensions other than three, functional analytic equations, and the profound connection with Jacobi theta functions and elliptic integrals; see [Glasser and Zucker 1980; Zucker 1984; Crandall 1996; Borwein and Borwein 1987]. It is an astounding fact that literally dozens of three-dimensional lattice sums are known in closed form, technically as Dirichlet $L$-series evaluations [Zucker 1987; 1990], yet none of these is quite the Madelung form (1.1). One of many examples is due to [Forrester and Glasser 1982], which says that if the origin for the lattice sum (1.1) is displaced by the vector $\vec{r}_{0}=\left(\frac{1}{6}, \frac{1}{6}, \frac{1}{6}\right)$, then the sum evaluates as $12^{s} L_{-4}(2 s-1)$, where here the $L$-series is defined by

$$
L_{-4}(t)=1^{-t}-3^{-t}+5^{-t}-7^{-t}+\cdots .
$$

In this particular variant, the physical case $s=\frac{1}{2}$ evaluates to $\sqrt{3}$, which is the exact potential at the point $\vec{r}_{0}$ within a full (sodium chloride) charge lattice. It is of interest that this potential is known exactly while $M$ remains elusive. In this regard, here is an amusing thought experiment. Remove the origin charge, in which case we know the potential at $\vec{r}_{0}$ to be exactly $\sqrt{3}-1 /\left|\vec{r}_{0}\right|=\sqrt{3}-\sqrt{12}=$ $-\sqrt{3} \sim-1.732$, which is within one per cent of the potential at the origin, displaced $\left(-\frac{1}{6},-\frac{1}{6},-\frac{1}{6}\right)$ away from $\vec{r}_{0}$ (this potential being $M$ itself). So in this approximate sense, we might say that $M$ "wants to be" minus the square root of three. There are other amusing equivalences, including this exact one: $M$ is precisely the electrostatic energy of a solitary charge residing at the center of a perfectly conducting unit cubical box [Crandall and Delord 1987].

We next embark on a brief tour of the computational developments that have sporadically emerged 
throughout the twentieth century. It has long been known that general Epstein zeta functions - in any number of dimensions - can be evaluated numerically via what might these days be called "fast" algorithms. The basic ideas go back to Riemann, and are relatively easy to apply in actual computations. An algorithm description from a modern perspective is [Crandall 1998b]. For crystal structures in particular, the Riemann approach gives rise to what is known in chemistry and physics as the Ewald expansion. For the function $M(s)$ of present concern, the Riemann formula takes the form of a rapidly convergent expansion:

$$
\begin{aligned}
\frac{\Gamma(s)}{\pi^{s}} M(s)=-\frac{1}{s} & +\sum_{x, y, z \in Z} \frac{\Gamma\left(s, \pi r^{2}\right)(-1)^{x+y+z}}{\left(\pi r^{2}\right)^{s}} \\
& +\sum_{k \in Z^{3}} \frac{\Gamma\left(\frac{3}{2}-s, \pi|k-c|^{2}\right)}{\left(\pi|k-c|^{2}\right)^{\frac{3}{2}-s}}
\end{aligned}
$$

where $r$ denotes the lattice radius $\sqrt{x^{2}+y^{2}+z^{2}}$, $c$ is the vector $\left\{\frac{1}{2}, \frac{1}{2}, \frac{1}{2}\right\}$, and $\Gamma(t, z)$ is the standard incomplete gamma function. The Ewald expansion is essentially the physical case $s=\frac{1}{2}$ of this rapidly convergent formula. Though only $O\left(\Delta^{3 / 2}\right)$ summands are required to yield $\Delta$ good digits, one generally needs evaluations of the incomplete gamma functions in one of the sums; in the $M$ computation one therefore needs $\Gamma\left(\frac{1}{2}, z\right)$, essentially the error function.

An era of less recondite, and perhaps more elegant, expansions for $M$ started in the 1950's with the Benson-Mackenzie formula, an attractive, twodimensional sum over positive odd integers:

$$
M=-12 \pi \sum_{x, y \in O^{+}} \operatorname{sech}^{2} \frac{\pi r}{2},
$$

where $r=\sqrt{x^{2}+y^{2}}$ is the two-dimensional lattice radius. Somewhat later, in 1975, emerged the Hautot formula:

$$
M=-\frac{\pi}{2}+3 \sum_{x, y \in Z}^{\prime} \frac{(-1)^{x} \operatorname{cosech}(\pi r)}{r} .
$$

The summands in both of these elegant formulae converge less rapidly - in the maximum lattice radius, say - than does the Ewald expansion, but of course these two younger series can be computationally superior, for two reasons: only elementary functions need be evaluated, and the sums are two-, not three-dimensional. Incidentally, there is a certain type of identity between Epstein zeta evaluations that typically leads to two-dimensional expansions. As discovered by [Zucker 1976; 1998] through adroit application of theta-function identities, it happens, nontrivially, that (letting $r$ denote the lattice radius $\sqrt{x^{2}+y^{2}+z^{2}}$ in the three-dimensional case)

$$
\begin{aligned}
M & =\frac{3}{\pi} \sum_{x, y, z \in Z}^{\prime} \frac{(-1)^{x+y}}{r^{2}} \\
& =\frac{3}{\pi} \sum_{x, y, z \in Z}^{\prime} \frac{(-1)^{x+y+z}}{x^{2}+2 y^{2}+2 z^{2}} \\
& =\frac{6}{\pi} \sum_{x, y, z \in Z}^{\prime} \frac{(-1)^{x}}{x^{2}+y^{2}+2 z^{2}}
\end{aligned}
$$

and this is by no means the end of the list of Zucker's equivalent forms. In addition, the standard functional equation for Epstein zeta functions gives

$$
M=\frac{4}{\pi} \sum_{x, y, z \in O}^{\prime} \frac{1}{r^{2}}
$$

a sum over all odd triples, the (not absolutely convergent) sum taken to be as usual the analytic continuation of $\sum^{\prime} r^{-2 s}$ to $s=1$. One only need Poissontransform any of the above right-hand sums - with respect to just one of the lattice indices - to introduce such decaying functions as cosech or coth, thereby arriving at rapidly convergent double sums for $M$. The first of Zucker's identities above, for example, quickly yields in this fashion the Hautot formula.

In [Crandall and Delord 1987] a two-dimensional series scheme for the "Madelung constant" of general crystal structures is derived, but the typical summand is rather intricate. In another treatment, [Crandall and Buhler 1987] exhibit a general prescription for casting any crystal structure's "Madelung constant" as a fairly simple series of elementary function evaluations (yet back to the threedimensional summation motif).

This brief overview of historical attempts brings us to an anecdote: a near-miss, if you will, in the quest for closed-form evaluation. In [Crandall and Buhler 1987] it is observed that one can come close to exact evaluations, in the sense that sufficiently 
acute knowledge of the asymptotic behavior of a certain sum over odd triples, namely

$$
S(t)=\sum_{u \in O^{3}} \frac{\operatorname{cosech}(\pi t|u|)}{|u|} \underset{t \rightarrow 0}{\sim} ?
$$

yields the Madelung constant as

$$
M=\lim _{t \rightarrow 0}\left(4 t S(t)-\frac{\pi}{2 t}\right) .
$$

This limit rule is by no means computationally vacuous - the authors were able to apply certain thetafunction identities as in [Glasser and Zucker 1980; Borwein and Borwein 1987; Zucker 1998], to arrive at values such as

$$
\begin{aligned}
& S\left(\frac{1}{\sqrt{8}}\right)=\sqrt{2} \theta_{2}^{2}\left(e^{-\pi / \sqrt{2}}\right)=\frac{\Gamma\left(\frac{1}{8}\right) \Gamma\left(\frac{3}{8}\right)}{2^{3 / 4} \pi^{3 / 2}}, \\
& S\left(\frac{1}{4}\right)=\sqrt{32} \theta_{2}\left(e^{-\pi \sqrt{2}}\right) \theta_{3}\left(e^{-\pi / \sqrt{2}}\right)=\frac{\Gamma\left(\frac{1}{8}\right) \Gamma\left(\frac{3}{8}\right) \sqrt{2}}{\pi^{3 / 2}},
\end{aligned}
$$

where $\theta_{2}, \theta_{3}$ are standard Jacobi theta functions. On the basis of the $t=\frac{1}{4}$ evaluation, the Madelung constant can be cast, through known connections between theta values and elliptic integrals, as a peculiar display of fundamental constants plus a final, minuscule, term:

$$
M=-2 \pi+\frac{\Gamma\left(\frac{1}{8}\right) \Gamma\left(\frac{3}{8}\right) \sqrt{2}}{\pi^{3 / 2}}+Y,
$$

where the constant $Y$ is a rapidly converging lattice sum

$$
Y=\sum_{x, y, z \in Z}^{\prime} \frac{(-1)^{x+y+z}}{r} \frac{2}{e^{4 \pi r}+1} .
$$

Because $Y$ is of order $e^{-4 \pi}$ (in fact, it is approximately $-0.00004152 \ldots$ ) we obtain five good decimals of $M$ even if $Y$ be ignored. One could say that $Y$ is the "Madelung constant" for a potential law of the short-range, or "Yukawa class," exhibiting exponential decay with distance as in nuclear physics models. In this sense, $M$ has been cast precisely in terms of another constant itself bestowed with a physical interpretation. It would be a grand achievement to evaluate $S(t)$ for even smaller $t$, thereby constricting the residual constant even further.

Any of these historical convergent series representations can be used to resolve hundreds - even thousands - of decimal digits of the Madelung constant. During computation, it is often required to evaluate either $r_{2}(n)$ or $r_{3}(n)$, where $r_{q}(n)$ is the number of representations of $n$ as a sum of $q$ squares.
The interesting computational details are discussed in [Buhler and Wagon 1996]. For the record, a Madelung value is

$$
\begin{aligned}
M= & M\left(\frac{1}{2}\right) \sim-1.747564594633182190636212 \backslash \\
& 03554439740348516143662474175815282 \ldots .
\end{aligned}
$$

In addition, one may use the incomplete-gamma expansion (1.2) to include analytic relatives of $M$ :

$$
\begin{aligned}
& M(1) \sim-2.51935615208944531334273065641 \ldots, \\
& M\left(\frac{3}{2}\right) \sim-3.2386247660517770978468809845 \ldots, \\
& M(2) \sim-3.8631638071965854864231037521 \ldots,
\end{aligned}
$$

$M(50) \sim-5.9999999999999893418589747421 \ldots$,

and so on. The $s=50$ instance reminds us that $M(s)$ for large $s$ is asymptotic to minus six, because, of course, there are six negative charges facing at unit distance in the defining sum (1.1). Such as the numerical values above are invaluable in the testing of any new computational scheme for $M(s)$.

The rest of the present treatment is reminiscent of the historical approaches, but stems from a relatively new theta-function identity and leads us accordingly in new directions. We shall heretofore concentrate not on the time-honored challenge of computational efficiency, rather on the introduction of new theoretical relations.

\section{ANALYTIC CONSEQUENCES OF THE ANDREWS IDENTITY}

All the new representations to follow originate in the beautiful identity of [Andrews 1986a; 1986b] for the cube of the Jacobi theta function $\theta_{4}$ :

$$
\begin{aligned}
\theta_{4}^{3}(q)= & \left(\sum_{n \in Z}(-1)^{n} q^{n^{2}}\right)^{3} \\
= & 1+4 \sum_{n=1}^{\infty} \frac{(-1)^{n} q^{n}}{1+q^{n}} \\
& -2 \sum_{n=1,|j|<n}^{\infty} \frac{q^{n^{2}-j^{2}}\left(1-q^{n}\right)(-1)^{j}}{1+q^{n}} .
\end{aligned}
$$

As Andrews points out, not every power of $q$ appears in the (expanded) right-hand side, because not every integer is a sum of three squares. Andrews also derived a cubic identity for sums of triangle numbers, from which identity one may infer the classical 
Gauss theorem that every natural number is a sum of three triangle numbers. We shall have more to say about sums of squares in a later section.

From one point of view, it has been the paucity of such cubic relations that explains the relatively low population of evaluations of Madelung and related constants in the physically meaningful, threedimensional settings. Conversely, exact evaluations for certain Epstein zeta values in 2, 4, 8 dimensions have arisen from long-known identities for powers $\theta_{4}^{2,4,8}$.

So, in pursuit of the Madelung constant, let us attempt a time-honored expedient, which is to use a theta-function identity within a Mellin transform. It is evident from the very structure of $\theta_{4}$ that (here and beyond we assume that $\operatorname{Re} s>0$ until otherwise specified):

$$
M(s)=\frac{1}{\Gamma(s)} \int_{0}^{\infty} t^{s-1}\left(\theta_{4}^{3}\left(e^{-t}\right)-1\right) d t .
$$

Inserting the Andrews identity (2.1) into this transform and rescaling the variable of integration results in

$$
\begin{aligned}
& M(s)=\frac{1}{\Gamma(s)} \sum_{n=1}^{\infty} \frac{1}{n^{s}} \int_{0}^{\infty} \frac{t^{s-1} d t}{1+e^{-t}} \\
& \quad \times\left(4(-1)^{n} e^{-t}-2\left(1-e^{-t}\right) \sum_{|j|<n}(-1)^{j} e^{-\left(n-j^{2} / n\right) t}\right) .
\end{aligned}
$$

It is useful at this juncture to introduce some nomenclature. The Lerch-Hurwitz eta function is taken to be

$$
\eta(s, a)=\sum_{k=0}^{\infty} \frac{(-1)^{k}}{(k+a)^{s}},
$$

where all through our treatment $a$ will be a positive real number. Immediate properties of $\eta$ include

$$
\begin{aligned}
\eta(s, 1) & =1^{-s}-2^{-s}+3^{-s}+\cdots \\
& =\left(1-2^{1-s}\right) \zeta(s), \\
\eta(s, a)+\eta(s, a+1) & =\frac{1}{a^{s}}
\end{aligned}
$$

and the integral representation

$$
\eta(s, a)=\frac{1}{\Gamma(s)} \int_{0}^{\infty} \frac{t^{s-1} e^{-a t} d t}{1+e^{-t}}
$$

which follows immediately by expanding $1 /\left(1+e^{-t}\right)$ in binomial series in $e^{-t}$, then integrating term-byterm. Equipped with these $\eta$-relations, we can cast (2.3) in the form

$$
\begin{aligned}
& M(s)=-4 \eta^{2}(s) \\
& \quad+2 \sum_{n \in Z^{+}} \sum_{|j|<n}(-1)^{j}\left(\frac{2}{\left(n^{2}-j^{2}\right)^{s}}-4 \frac{\eta\left(s, n-j^{2} / n\right)}{n^{s}}\right) .
\end{aligned}
$$

Now we embark on an intricate reduction task, whose steps include separating off the $j=0$ terms, pairing the \pm instances of $j$, introducing an extra summation index for the final $\eta$ function in (2.4), and some reindexing/rearrangement. This procedure results in a representation

$$
M(s)=-6 \eta^{2}(s)-4 S(s)-8 T(s),
$$

where we define

$$
\begin{aligned}
& S(s)=\sum_{n>j} \frac{(-1)^{j}}{\left(n^{2}-j^{2}\right)^{s}}, \\
& T(s)=\sum_{m>n>j} \frac{(-1)^{m+n+j}}{\left(m n-j^{2}\right)^{s}},
\end{aligned}
$$

with all summation indices for both $S$ and $T$ deemed positive. Happily, perhaps surprisingly, the $S$ function admits of exact evaluation. Write

$$
\begin{aligned}
S(s) & =\sum_{n>m} \frac{(-1)^{m+n}}{(2 n-m)^{s} m^{s}} \\
& =-\frac{1}{2} \zeta(2 s)+\frac{1}{2} \sum_{m<2 n} \frac{(-1)^{m+n}}{(2 n-m)^{s} m^{s}} .
\end{aligned}
$$

Now the relevant sum is a convolution, and we might expect simplification. Indeed, breaking up this convolution into even- $m$ and odd- $m$ parts, it is not hard to establish the closed form

$$
S(s)=\frac{1}{2}\left(2^{-2 s} \eta^{2}(s)+\beta^{2}(s)-\zeta(2 s)\right),
$$

where $\beta$ is another standard $L$-series, $\beta(s)=1^{-s}-$ $3^{-s}+5^{-s}-7^{-s}+\cdots$, so that representation $(2.5)$ is not the last word. Inserting our closed-form $S$, we get

$$
\begin{aligned}
M(s)=-\left(6+2^{1-s}\right) \eta^{2}(s) & \\
& -2 \beta^{2}(s)+2 \zeta(2 s)-8 T(s),
\end{aligned}
$$

of which only the $T$ function stands in the way of what might be called a complete evaluation of $M(s)$. Incidentally, the apparent pole at $s=\frac{1}{2}$ in the $\zeta$ 
function is specious; a cancellation can be extracted from within the $T$ function, as we shall see.

There is a fascinating, alternative line of analysis, starting with (2.5) but thinking of $T$ in the form

$$
T(s)=\sum_{a>b} \frac{(-1)^{a+b+j}}{\left((a+j)(b+j)-j^{2}\right)^{s}},
$$

where all implicit summation indices $a, b, j$ are positive. An immediate relation is then

$$
2 T(s)+S(s)=U(s),
$$

where the new function $U$ is defined by

$$
U(s)=\sum \frac{(-1)^{x+y+z}}{(x y+y z+x z)^{s}},
$$

in which all implicit summation indices $x, y, z$ are deemed positive. Accordingly, a compact companion representation to $(2.5),(2.6)$ is

$$
M(s)=-6 \eta^{2}(s)-4 U(s) .
$$

One might remark that yes, either (2.6) or (2.7) has just one remaining function to evaluate, but these new functions $T, U$ involve triple sums over quadratic-form denominators, and so little is gained. That remark would be well taken, except that these new representations give rise to connections with several other fields of analysis, as we shall see. In particular, the quadratic form $x y+y z+x z$ within the $U$ function is linear with respect any one coordinate, and this brings considerable advantage during analysis. For another thing, the indicial constraints in the $T$ sum lead naturally to a connection with what are called multiple zeta sums.

\section{CONNECTION WITH MULTIPLE ZETA SUMS}

Relation (2.6) motivates us to establish representations for the $T$ function. It is desirable to cancel the specious singularity $(\zeta(1))$ in $(2.6)$, and we shall be able to do so in what follows. From the definition

$$
T(s)=\sum_{m>n>j} \frac{(-1)^{m+n+j}}{\left(m n-j^{2}\right)^{s}}
$$

we contemplate a formal binomial expansion for the $(-s)$-th power:

$$
T(s)=\sum_{\mu=0}^{\infty}\left(\begin{array}{c}
-s \\
\mu
\end{array}\right)(-1)^{\mu} \zeta^{--}(s+\mu, s+\mu,-2 \mu) .
$$

where the triple sum is defined

$$
\zeta^{---}(a, b, c)=\sum_{m>n>j} \frac{(-1)^{m+n+j}}{m^{a} n^{b} j^{c}},
$$

being a particular, three-dimensional instance of a generalized "multiple zeta sum" denoted

$$
\zeta^{ \pm \pm \cdots}\left(s_{1}, s_{2}, \ldots\right)=\sum_{n_{1}>n_{2}>\cdots} \frac{( \pm 1)^{n_{1}}}{n_{1}^{s_{1}}} \frac{( \pm 1)^{n_{2}}}{n_{2}^{s_{2}}} \cdots
$$

Now, these multiple zeta sums have enjoyed considerable modern interest. The study of such sums goes back to Euler, who evaluated certain instances algebraically. Much research of today is concerned with open conjectures and new results, some of which obtainable through sheer numerical experiment (and then to be proven, if possible, later). The notion of mixing theory and experiment in the quest for exact evaluations has been carried out in spectacular fashion by a group of researchers, as in [Bailey et al. 1994]. The sums enjoy connections with knot theory and with high-energy physics, such connections having been pioneered by D. J. Broadhurst and colleagues. The literature is already diverse, ranging across the numerical-theoretical spectrum [Crandall and Buhler 1994; Crandall 1998a; Borwein et al. 1998b]. There is actually an elegant algebraic treatment of triple sums in particular [Borwein and Girgensohn 1996]. That treatment concerns multiple zetas with positive integer arguments, and reveals that $\zeta^{+++}(a, b, c)$ is reducible to lower-dimensional zetas if $a+b+c$ be even. The methods therein may well apply in our present case, where $c$ will be zero or negative as in (3.1).

The $\mu=0$ term in the expansion (3.1) is special for various reasons, so we analyze it first. We have

$$
\begin{aligned}
\zeta^{---}(s, s, 0) & =\sum_{m>n} \frac{(-1)^{m+n}}{m^{s} n^{s}} \sum_{j<n}(-1)^{j} \\
& =-\frac{1}{2} \sum_{m>n} \frac{(-1)^{m+n}}{m^{s} n^{s}}\left(1+(-1)^{n}\right) \\
& =-\frac{1}{2} \zeta^{--}(s, s)-\frac{1}{2} \zeta^{-+}(s, s) .
\end{aligned}
$$

Thus the triple zeta sum in question reduces to double sums. From a symmetry argument (actually, swapping summation indices at the right moment) it is not hard to see that

$$
\zeta^{--}(s, s)=-\frac{1}{2}\left(\zeta^{2}(2 s)-\eta^{2}(s)\right)
$$


and we conclude

$$
\zeta^{---}(s, s, 0)=-\frac{1}{4} \eta^{2}(s)+\frac{1}{4} \zeta(2 s)-\frac{1}{2} \zeta^{-+}(s, s) .
$$

Putting all of this together with (2.6) we have a representation

$$
\begin{gathered}
M(s)=-\left(4+2^{1-s}\right) \eta^{2}(s)-2 \beta^{2}(s)+4 \zeta^{-+}(s, s) \\
-8 \sum_{\mu=1}^{\infty}\left(\begin{array}{c}
-s \\
\mu
\end{array}\right)(-1)^{\mu} \zeta^{---}(s+\mu, s+\mu,-2 \mu) .
\end{gathered}
$$

Now, on the assumption that the $\zeta^{-+}$term is a regular function - admittedly an unproven assumption in our treatment - there is no evident singularity in the $s$-plane because the one-dimensional $\zeta$ has been effectively removed from (2.6). As to questions of convergence, we can say that (3.2) converges absolutely for sufficiently large Res, on the basis of growth estimates for the triple zeta sum. The issue of precise domain of convergence for the $\mu$ series is open.

An intriguing special case of (3.2), namely $s=1$ (not the Madelung case) enjoys a certain "serial evaluation" property: every summand will admit of exact evaluation. Because $\zeta^{-+}(1,1)$ can be evaluated easily (via partial fraction decomposition, say) we can use the result

$$
\zeta^{-+}(1,1)=\frac{1}{2} \log ^{2} 2
$$

to obtain

$$
\begin{aligned}
M(1)=-\frac{1}{8} \pi^{2} & -\frac{5}{2} \log ^{2} 2 \\
& -8 \sum_{\mu=1}^{\infty} \zeta^{---}(\mu+1, \mu+1,-2 \mu) .
\end{aligned}
$$

Now we argue that every triple zeta sum in (3.3) can be evaluated. Take $\mu=1$ as example, in which case

$$
\begin{aligned}
\zeta^{--}(2,2,-2) & =\sum_{m>n} \frac{(-1)^{m+n}}{m^{2} n^{2}} \sum_{j<n} j^{2} \\
& =-\frac{1}{2} \sum_{m>n} \frac{(-1)^{m+n}}{m^{2} n^{2}}(-1)^{n}\left(n^{2}-n\right) \\
& =-\frac{1}{2}\left(-\eta(1)+\eta(2)-\zeta^{-+}(2,1)\right) .
\end{aligned}
$$

But we are not stuck here, for the double zeta sum in question has been evaluated. One may use either the algebraic approach of [Bailey et al. 1994] or the integral representation theory of [Crandall and Buhler 1994] to prove

$$
\zeta^{-+}(2,1)=\frac{1}{8} \zeta(3) .
$$

Therefore the first summand in (3.3) is

$$
\begin{aligned}
\zeta^{---}(2,2,-2) & =-\frac{1}{24} \pi^{2}+\frac{1}{2} \log 2+\frac{1}{16} \zeta(3) \\
& \sim 0.0104 \ldots
\end{aligned}
$$

In like manner, one can obtain the next $(\mu=2)$ summand of (3.3) as

$$
\begin{aligned}
\zeta^{---} & (3,3,-4) \\
& =-\frac{5}{48} \pi^{2}+\frac{1}{4} \log 2+\frac{3}{4} \zeta(3)+\frac{5}{96} \pi^{2} \zeta(3)-\frac{41}{64} \zeta(5) \\
& \sim 0.000371 \ldots .
\end{aligned}
$$

One may continue this procedure ad infinitum; in fact the "serial evaluation" property enjoyed by the $M(1)$ expansion is that the $\mu$-th summand can be evaluated if $\zeta^{--}(\mu+1, \mu+1,-2 \mu)$ can, and this in turn follows from evaluation of $\zeta^{-+}(\mu+1, \mu)$, as above for $\mu=1,2$. The final part of the argument is that $\zeta^{-+}$can be evaluated when the "odd-weight rule" is in effect; i.e., the sum of the two integer arguments is odd, as $2 \mu+1$ is.

During analysis of our particular triple zeta sums, we require sums of $(-1)^{j} j^{2 \mu}$ for $j \in[1, \ldots, n-1]$. We observe that for $\mu=0$ this sum is $-\left(1+(-1)^{n}\right) / 2$, and then generally, for integers $\mu>0$, is

$$
\sum_{j<n}(-1)^{j} j^{2 \mu}=-\frac{(-1)^{n}}{2} E_{2 \mu}(n),
$$

where $E_{k}$ is the Euler polynomial of index $k$. It follows from this identity that the left-hand sum is a polynomial of degree $2 \mu$ in $n$, and except for the highest term all other powers are odd powers of $n$. It is this very last property that allows certain of the $\zeta^{---}$to be evaluated on the basis of the odd-weight rule.

There may well be other $s$ for which each summand of (3.2) admits evaluation in the above style. However, some difficulty can certainly be expected, given the following evidence. For the case $s=2$, we expect to evaluate $\zeta^{-+}(2,2)$ at some point, yet this can be achieved through some results of [Bailey et al. 1994], namely

$$
\begin{aligned}
\zeta^{-+}(2,2)=\frac{13}{288} \pi^{4} & +\frac{1}{6} \pi^{2} \log ^{2} 2 \\
& -\frac{1}{6} \log ^{4} 2-\frac{7}{2} \zeta(3) \log 2-4 \operatorname{Li}_{4}\left(\frac{1}{2}\right),
\end{aligned}
$$

which is certainly a stultifying expression, what with appearance of the polylogarithm function $\mathrm{Li}_{4}$. What 
is more, those authors argue, on the basis of extensive numerical evidence, that certain double zeta sums probably cannot be written in terms of $\zeta$ series, even with polylogarithm components. Thus it may turn out that $s$ values for which serial evaluation is possible, summand by summand, are sparse.

\section{AN $\eta$-SERIES FOR $M(s)$}

Observations of the previous section notwithstanding, it is possible to exhibit a different series for $M(s)$ that patently adheres to the serial evaluation principle, that is, every series term can be given an exact evaluation. Each term will in fact be a superposition of finitely many $\eta$ values.

We have seen, as in relation $(2.7)$, that $M(s)$ can be resolved whenever

$$
U(s)=\sum \frac{(-1)^{x+y+z}}{(x y+y z+x z)^{s}}
$$

can be. We proceed formally, without regard to convergence issues as yet, invoking the particular binomial expansion

$$
\begin{aligned}
U(s) & =2^{s} \sum \frac{(-1)^{x+y+z}}{\left((x+y+z)^{2}-x^{2}-y^{2}-z^{2}\right)^{s}} \\
& =2^{s} \sum_{\mu=0}^{\infty}\left(\begin{array}{c}
-s \\
\mu
\end{array}\right)(-1)^{\mu} \sum_{n=1}^{\infty} \frac{(-1)^{n} F(n, \mu)}{n^{2 s+2 \mu}},
\end{aligned}
$$

where we introduce a combinatorial function

$$
F(n, \mu)=\sum_{x+y+z=n}\left(x^{2}+y^{2}+z^{2}\right)^{\mu} .
$$

We observe that $F$ is generally a polynomial of degree $2 \mu+2$ in $n$, so we proceed to define coefficients implicitly as

$$
F(n, \mu)=\sum_{k=0}^{2 \mu+2} f_{\mu k} n^{k}
$$

For example,

$$
F(n, 2)=\frac{n}{5}+\frac{n^{2}}{30}-n^{3}+\frac{4 n^{4}}{3}-\frac{7 n^{5}}{10}+\frac{2 n^{6}}{15},
$$

which shows the degree, and the tendency for all powers except constant term to appear in $F$. In fact, $f_{\mu 0}=0$ for all $\mu>0$, but one must take care during analysis, since $F(n, 0)=1-\frac{3}{2} n+\frac{1}{2} n^{2}$.
The $f_{\mu k}$ coefficients can be inserted directly to yield an expansion for $M(s)$, which we call the " $\eta$ series":

$$
M(s)=-6 \eta^{2}(s)+2^{s+2} \sum_{\mu=0}^{\infty} W(\mu, s),
$$

with the assignment

$$
W(\mu, s)=\left(\begin{array}{c}
-s \\
\mu
\end{array}\right)(-1)^{\mu} \sum_{k=0}^{2 \mu+2} f_{\mu k} \eta(2 s+2 \mu-k),
$$

which is manifestly a finite superposition of $\eta$ values. That the $W$ summands are rational (when $s$ is) follows from the fact that the $f_{\mu k}$ can be writtenalbeit in a complicated way - in terms of Bernoulli numbers. We remind ourselves that the $\eta$ series (4.1) is merely formal. However, on the basis of numerical experiments it is natural to conjecture that, ordered as written (i.e., initialize $\mu=0$, perform the $k$ sum, then advance $\mu$, and so on) this $\eta$ series converges for all complex $s$. Such conjecture needs be stated in this way, because other tempting reorderings may not converge. For example, one looks longingly at the $\eta$ series and considers, say, collecting all terms in $\eta(t)$ for fixed $t$. It turns out, unfortunately, that the coefficient of such a fixed $\eta$ diverges in general. However, to exemplify the conjectured convergence of (4.1) as ordered, note that the example of Madelung constant $M=M\left(\frac{1}{2}\right)$ has the following series terms, for cases $\mu=0,1,2$ respectively of the $\mu$ summand:

$$
\begin{aligned}
W\left(0, \frac{1}{2}\right) & =-\frac{5}{8}+\log 2 \sim 0.0681 \ldots \\
W\left(1, \frac{1}{2}\right) & =-\frac{7}{32}-\frac{1}{48} \pi^{2}+\frac{5}{8} \log 2 \sim 0.00885 \ldots, \\
W\left(2, \frac{1}{2}\right) & =-\frac{19}{160}-\frac{1}{32} \pi^{2}+\frac{7}{9600} \pi^{4}+\frac{1}{2} \log 2+\frac{3}{320} \zeta(3) \\
& \sim 0.00169 \ldots
\end{aligned}
$$

The precise rate of convergence is unknown, but the value for the sum over $\mu \in[0,15]$ is

$$
M \sim-1.747564597 \ldots,
$$

correct to nine good decimals.

It is an interesting exercise to show from the $\eta$ series the known analytic behavior that $M(n)$ vanishes for all negative integers $n$; for such arguments the $\mu$ sum terminates. Even simpler is to establish the known analytic continuation value $M(0)$, as there 
is only one term in the $\mu$ sum, and we obtain the known evaluation

$$
M(0)=-6 \eta^{2}(0)+4 \eta(0)-6 \eta(-1)+2 \eta(-2)=-1 .
$$

\section{INTEGRAL REPRESENTATIONS FOR THE MADELUNG CONSTANT}

We have seen that

$$
\begin{aligned}
M(s) & =-6 \eta^{2}(s)-4 U(s) \\
& =-6 \eta^{2}(s)-4 \sum \frac{(-1)^{x+y+z}}{(x y+y z+x z)^{s}},
\end{aligned}
$$

which expression allows interesting integration procedures, at least in the physical case $s=\frac{1}{2}$. Observe first that, from the aforementioned integral representation for the $\eta$ function, $\eta^{2}$ can be cast as an integral over the two-dimensional plane $R^{2}$, and for $s=\frac{1}{2}$ the integration goes:

$$
\begin{aligned}
\eta^{2}\left(\frac{1}{2}\right) & =\frac{1}{\pi} \int_{0}^{\infty} \int_{0}^{\infty} \frac{d t}{\sqrt{t}} \frac{d u}{\sqrt{u}} \frac{1}{e^{t}+1} \frac{1}{e^{u}+1} \\
& =\frac{1}{\pi} \int_{R^{2}} \frac{d t d u}{\left(e^{t^{2}}+1\right)\left(e^{u^{2}}+1\right)} .
\end{aligned}
$$

Surprisingly enough, the remaining piece $U\left(\frac{1}{2}\right)$ also admits of an area-integral representation. Observe first that

$$
\int_{R^{2}} d t d u e^{-t^{2}(x+z)-u^{2}(y+z)+2 u t z}=\frac{\pi}{\sqrt{x y+y z+x z}} .
$$

Then we exploit the unusual quadratic form $x y+$ $y z+x z$ whose origin lies in the Andrews identity. Summation over the full $(x, y, z)$ lattice is now (formally) possible within the integral, and we obtain

$$
U\left(\frac{1}{2}\right)=-\frac{1}{\pi} \int_{R^{2}} \frac{d t d u}{\left(e^{t^{2}}+1\right)\left(e^{u^{2}}+1\right)\left(e^{(t-u)^{2}}+1\right)} .
$$

Using these area integrals, and changing to rootpolar coordinates $(\sqrt{\rho}, \theta)$, we obtain the Madelung constant as

$M=\frac{1}{2 \pi} \int_{0}^{\infty} d \rho \int_{0}^{2 \pi} d \theta \frac{-6+4\left(1+e^{\rho(\cos \theta-\sin \theta)^{2}}\right)^{-1}}{\left(1+e^{\rho \cos ^{2} \theta}\right)\left(1+e^{\rho \sin ^{2} \theta}\right)}$.

If one insists on a finite domain, then setting $\rho=$ $-\log r$ results in a finite integral representation, a certain average value over the unit disk:

$$
M=\frac{1}{2 \pi} \int_{0}^{1} d r \int_{0}^{2 \pi} d \theta \frac{-6+4\left(1+r^{-(\cos \theta-\sin \theta)^{2}}\right)^{-1}}{\left(1+r^{\cos ^{2} \theta}\right)\left(1+r^{\sin ^{2} \theta}\right)} .
$$

Though these results may only be of theoretical interest, the integrands are well-behaved; nothing prevents one from performing the finite-area integral, say, to obtain a reasonable numerical value.

Incidentally, there are yet other ways to arrive at integral representation for $U(s)$. One approach is to write

$$
\begin{aligned}
U(s) & =\sum \frac{(-1)^{x+y+z}}{(x y z)^{s}} \frac{1}{(1 / x+1 / y+1 / z)^{s}} \\
& =\frac{1}{\Gamma(s)} \int_{0}^{\infty} t^{s-1} \chi^{3}(s, t) d t,
\end{aligned}
$$

where $\chi$ is the peculiar sum

$$
\chi(s, t)=\sum_{n=1}^{\infty} \frac{(-1)^{n}}{n^{s}} e^{-t / n},
$$

which differs from typical theta functions in the form of the exponent (witness the inversion of $n$ ). Another method is to cast (5.1) in the form of a spectral integral:

$$
U\left(\frac{1}{2}\right)=-\frac{1}{\pi^{2}} \int_{0}^{\infty} f^{3}(\omega) d \omega,
$$

where $f$ is the Fourier transform

$$
f(\omega)=\int_{-\infty}^{\infty} \frac{e^{i \omega t} d t}{e^{t^{2}}+1} .
$$

Similar but more complicated spectral integrals can be achieved for general $s$.

It is evidently difficult to derive from these integral representations a general, fast computational algorithm - in the style of the Riemann-Ewald formula (1.2) say - for nontrivial $M(s)$ values. It happens, however, that an elementary-function expansion can be obtained whenever $s$ is half an odd integer. Take the example $s=\frac{1}{2}$, for which we may, by analogy with standard Poisson transformation of theta functions, obtain the following form for the $\chi$ function:

$$
\chi\left(\frac{1}{2}, t\right)=\sqrt{i} \sum_{d \in O} \frac{e^{-\gamma \sqrt{2 \pi d t}}}{\sqrt{d}},
$$

where $\gamma=1-i$, the sum being taken over all odd integers $d$, with negative $d$ handled according to the branch rule $\sqrt{-|d|}:=i \sqrt{|d|}$. Then in (5.4) we partition the domain of integration into intervals $(0, \lambda)$ 
and $(\lambda, \infty)$, using the form (5.6) for the second interval only, to get a convergent representation:

$$
\begin{aligned}
U\left(\frac{1}{2}\right) & =-\frac{1}{\sqrt{\pi}} \sum_{N=0}^{\infty}\left(\frac{(-1)^{N} \lambda^{N+1 / 2}}{N+1 / 2}\right. \\
& \left.\times \sum_{a+b+c=N} \frac{\eta\left(a+\frac{1}{2}\right) \eta\left(b+\frac{1}{2}\right) \eta\left(c+\frac{1}{2}\right)}{a ! b ! c !}\right) \\
- & \frac{1}{\pi} \sum_{m, n, p \in O} \frac{e^{-\gamma(\sqrt{m}+\sqrt{n}+\sqrt{p}) \sqrt{2 \pi \lambda}}}{\sqrt{m} \sqrt{n} \sqrt{p}(\sqrt{m}+\sqrt{n}+\sqrt{p})},
\end{aligned}
$$

with the $a, b, c$ indices each nonnegative and the aforementioned neagtive-radicand rule in effect. In this way we have (via the pathway of the Andrews identity) at least one rapidly convergent expansion for the Madelung constant $M=-6 \eta^{2}\left(\frac{1}{2}\right)-4 U\left(\frac{1}{2}\right)$. It is not too troublesome to obtain a hundred or so digits of precision in this manner, say for $\lambda=1$. The free parameter $\lambda$ is a powerful practical tool: changing the value of $\lambda$ must, in practice, yield an invariant result. Finally, we address the claim that halfodd $s$ allow elementary-function expansions. For general $s$ one may proceed by establishing a Poisson form such as (5.6), but involving values of the Hankel-Bessel function $H_{s-1}$, which, for any halfodd value $s \in\left\{\frac{1}{2}, \frac{3}{2}, \frac{5}{2}, \frac{7}{2}, \ldots\right\}$, is elementary.

\section{NUMBER-THEORETICAL IMPLICATIONS: SUMS OF THREE SQUARES}

The representation (2.7) can be thought of as an analytic form of a modified Andrews identity, which variant we now state as

$$
\begin{aligned}
\theta_{4}^{3}(q)=1-6 \sum & (-1)^{x+y} q^{x y} \\
& -4 \sum(-1)^{x+y+z} q^{x y+y z+x z}
\end{aligned}
$$

where all summation indices are positive. This identity can be obtained via indicial manipulations of the original identity (2.1); but truth is, this new form (6.1) was first found by the author in the context of the Madelung problem. We can write the essential content of the new identity as an expression for $r_{3}(N)$, the number of representations of $N$ as a sum of three squares. For $N>1$ we obtain

$$
\begin{aligned}
(-1)^{N} r_{3}(N)=-6 \sum_{x y=N}(-1)^{x+y} & \\
& -4 \sum_{x y+y z+x z=N}(-1)^{x+y+z},
\end{aligned}
$$

where every summation index $x, y$ or $z$ is deemed positive. The first term on the right hand side, thought of as arising from an analytic form $-6 \eta(s)^{2}$, can be analyzed by observing that

$$
\eta^{2}(s)=\left(1-2^{2-s}+2^{2-2 s}\right) \zeta^{2}(s),
$$

so that

$$
\sum_{x y=N}(-1)^{x+y}=d(N)-4 d(N / 2)+4 d(N / 4)=\Delta(N),
$$

where the divisor function $d(m)$ is defined here as the number of divisors of $n$, except $d(m)=0$ if $m$ is not a positive integer. Note that for odd $f$ we have the doubling formula $d\left(2^{a} f\right)=(a+1) d(f)$. If $N$ be decomposed into even/odd factors, we arrive at these results:

Lemma 1. Let a positive integer $N=2^{a} f$ with $f$ odd. If $N$ be odd then $\Delta(N)=d(N)$, while for $N$ even we have

$$
\Delta(N)=(a-3) d(f) .
$$

Lemma 2. The representation number $r_{3}(N)$ can be written, for the decomposition $N=2^{a} f$ with $f$ odd, as

$$
\begin{aligned}
(-1)^{N} r_{3}(N)=-6(a-3+ & \left.4 \delta_{a 0}\right) d(f) \\
& -4 \sum_{x y+y z+x z=N}(-1)^{x+y+z} .
\end{aligned}
$$

In particular, the coefficient of $d(f)$ vanishes if and only if $N$ is an odd multiple of eight, in which cases $r_{3}(N)$ is a multiple of four.

The intriguing analytic phenomenon behind the last sentence in Lemma 2 is that if one squares the $\eta$ function $1 / 1^{s}-1 / 2^{s}+1 / 3^{s}-\cdots$ symbolically, and regroups into a Dirichlet series, then the coefficients of $1 / 8^{s}, 1 / 24^{s}, 1 / 40^{s}, \ldots$ are the only vanishing ones.

It has long been known that $N$ is a sum of three squares if and only if $N$ is not of the exceptional form $4^{b}(8 k+7)$. Since $N=8 f$ with $f$ odd can never be of that form, Lemma 2 reveals that the $x, y, z$ sum cannot be empty for such an $N$, and we have proved:

Theorem 1. Every positive integer can be represented as $x^{2}+y^{2}+z^{2}$ or $x y+y z+x z$.

An explicit example of the workings of the theorem is this: 1308732 is not a sum of three squares, yet $1308732=426 \cdot 950+426 \cdot 657+950 \cdot 657$. On the 
other hand, $78=5^{2}+7^{2}+2^{2}$ cannot be written in the form $x y+y z+x z$. Though many integers enjoy both types of representation, it is - perhaps surprisingly - the case that the $x y+y z+x z$ form is more common, in the following sense [Borwein and Choi 2000]. It can be shown by class field theory that only finitely many integers cannot be represented $x y+y z+x z$, with $x, y, z>0$. Specifically, the exceptions are: $1,4,2,6,10,18,22,30,42,58,70$, $78,102,130,190,210,330,462$, in addition to at most one more exceptional value that would have to be larger than $10^{11}$. The idea of the proof is that a square-free $N$ is not represented if and only if $-N$ is a disjoint discriminant (i.e., the complex quadratic field $Q(\sqrt{-N})$ has exactly one ideal class in each genus). It is known that if the generalized Riemann hypothesis (GRH) is true then there is no such exceptional $N>10^{11}$. In any case either 18 or 19 total instances are not representable. In particular, this line of argument settles in the positive a previous conjecture of the present author, that every odd integer $N>1$ is representable. This result may seem to deflate somewhat our Theorem 1, but of course the present approach, based as it is on the Andrews identity, does not rely upon the intricacies of class field theory.

Such results as the modified theta-function identity, subsequent Theorem, and observations from class field theory compel us to analyze sums over $x y+y z+x z=N$ in earnest. We observe a combinatorial decomposition, written in operator form as

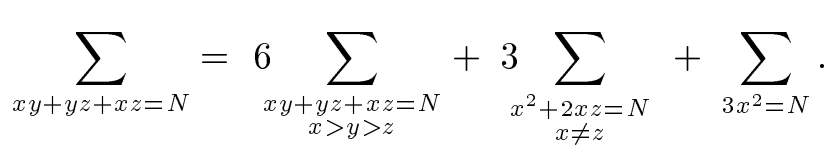

Thus for example the final sum vanishes unless $N$ is three time a square, the penultimate sum is twodimensional, and in the analytic mode we are moved to consider functions such as

$$
V(s)=\sum_{x>y>z} \frac{(-1)^{x+y+z}}{(x y+y z+x z)^{s}}
$$

It can be expected that representations based on this function converge, due to the extra indicial constraints, somewhat better than those based on the
$U$ function. For example, the Madelung constant might be cast in terms of an integral:

$V\left(\frac{1}{2}\right)=-\frac{1}{\pi} \int_{R^{2}} \frac{d t d u}{\left(e^{t^{2}}+1\right)\left(e^{t^{2}+u^{2}}+1\right)\left(e^{t^{2}+u^{2}+(t-u)^{2}}+1\right)}$,

which may well exhibit superior convergence properties when compared to previous integrals.

\section{OPEN PROBLEMS}

A computational issue is: can integral representations of Sections 5,6 be used in a computational algorithm for general arguments $s$, of efficiency competing, say, with the historical methods of Section 1 ? In some integral representation involving a term

$$
\left(e^{x}+1\right)^{-1}
$$

one might insert the known Bernoulli expansion

$$
\frac{1}{e^{x}+1}=-\sum_{n=1}^{\infty} \frac{2^{n}-1}{n !} B_{n} x^{n-1} .
$$

Note that this Bernoulli decomposition is only valid for $|x|<\pi$, so that we still would need a second integral over larger $x$ values.

Another question is: can either the triple-zeta series (3.2) or the $\eta$ series (4.1) be reordered with a view to simplicity? With a view to faster convergence? We remind ourselves of the conjecture that the $\eta$ series converges as written, for all complex $s$; which conjecture is so far based on numerical evidence.

Noting that the $U$ function appearing in (2.7) is a variant of Epstein zeta functions, in that a quadratic form $x y+y z+x z$ appears, we expect some manner of functional equation exists for $U$. One could fuse together the established functional equations for $M$ and $\eta$. The question would be, what information - about quadratic-form representations, perhaps - could be gleaned from such a functional equation?

As for number-theoretical questions per se, can an efficient algorithm for computation of $r_{3}(N)$ make use of the new variant of the Andrews identity, in the guise of Lemma 2, Section 6? One promising avenue would be the establishment of recursion relations attendant to the quadratic form $x y+y z+x z$.

We conclude by indicating yet another intriguing arena of potential applications for the Andrews 
identity: lattice-sum estimation. For example, consider the three-dimensional variant of the celebrated Gauss "circle problem," which variant is to bound rigorously the fluctuation of $\left|\#(r)-4 \pi r^{3} / 3\right|$, where $\#(r)$ is the number of lattice points within a sphere of radius $r$. If we denote

$$
Z(s)=\sum_{x, y, z \in Z}^{\prime} \frac{1}{\left(x^{2}+y^{2}+z^{2}\right)^{s}},
$$

then for real $r>0, r^{2}$ not an integer, the latticepoint count is given exactly by such representations as

$$
\#(r)=\frac{1}{2 \pi i} \int_{(\sigma)} r^{2 s} Z(s) \frac{d s}{s},
$$

where the contour integral is along the line $s=\sigma+$ $i \tau$, with $\sigma>\frac{3}{2}$ fixed. It is possible that the Andrews identity, when applied to such representations (with $q$ replaced by $-q$ ), would yield error terms of a new form, and perhaps of unprecedented tractability.

\section{ACKNOWLEDGMENTS}

The author is grateful to I. Zucker, J. Borwein, D. Bradley for their insight and results in regard to lattice sums; and to N. Wheeler for his expertise on the history and formalism of the Madelung problem. The author acknowledges valuable pedagogical discussions with T. Dunne. Colleagues S. Wolfram, V. Adamchik, and S. Wagon provided key algebraic and computational observations. The author thanks an astute referee for outlining the class field theory argument following Theorem 1. Without the benefit of a fascinating conversation in the early 1990's with G. Andrews and J. Buhler, the author would have had, along the lines of this paper, nothing to say.

\section{REFERENCES}

[Andrews 1986a] G. E. Andrews, "The fifth and seventh order mock theta functions", Trans. Amer. Math. Soc. 293:1 (1986), 113-134.

[Andrews 1986b] G. E. Andrews, "EฯPHKA! num = $\Delta+\Delta+\Delta "$, J. Number Theory 23:3 (1986), 285-293.

[Bailey et al. 1994] D. H. Bailey, J. M. Borwein, and R. Girgensohn, "Experimental evaluation of Euler sums", Experiment. Math. 3:1 (1994), 17-30.

[Borwein and Borwein 1987] J. M. Borwein and P. B. Borwein, $P i$ and the AGM: A study in analytic number theory and computational complexity, Wiley, New York, 1987.

[Borwein and Borwein 1994] D. Borwein and J. M. Borwein, "On some trigonometric and exponential lattice sums", J. Math. Anal. Appl. 188:1 (1994), 209218.

[Borwein and Choi 2000] J. Borwein and K.-K. S. Choi, "On the representations of $x y+y z+z x$ ", Experimental Mathematics 9 (2000). See http://www.cecm.sfu.ca/ preprints/.

[Borwein and Girgensohn 1996] J. M. Borwein and R. Girgensohn, "Evaluation of triple Euler sums", Electron. J. Combin. 3:1 (1996), Res. Paper 23.

[Borwein et al. 1985] D. Borwein, J. M. Borwein, and K. F. Taylor, "Convergence of lattice sums and Madelung's constant", J. Math. Phys. 26:11 (1985), 2999-3009.

[Borwein et al. 1998a] D. Borwein, J. M. Borwein, and C. Pinner, "Convergence of Madelung-like lattice sums", Trans. Amer. Math. Soc. 350:8 (1998), 3131-3167.

[Borwein et al. 1998b] J. Borwein, D. Bradley, D. Broadhurst, and P. Lisonek, "Special values of multidimensional polylogarithms", Research Report 98-106, CECM, Simon Fraser University, Burnaby, BC, 1998. See http://www.cecm.sfu.ca/preprints/. Version $\aleph_{0}$.

[Buhler and Crandall 1990] J. P. Buhler and R. E. Crandall, "On the convergence problem for lattice sums", J. Phys. A Math. Gen. 23:12 (1990), 25232528.

[Buhler and Wagon 1996] J. Buhler and S. Wagon, "Secrets of the Madelung constant", Mathematica in Education and Research 5:2 (1996), 49-55.

[Crandall 1996] R. E. Crandall, Topics in advanced scientific computation, Springer, New York, 1996.

[Crandall 1998a] R. E. Crandall, "Fast evaluation of multiple zeta sums", Math. Comp. 67:223 (1998), 1163-1172.

[Crandall 1998b] R. Crandall, "Fast evaluation of Epstein zeta functions", preprint, 1998. See http:// www.perfsci.com/free/techpapers/index.html.

[Crandall and Buhler 1987] R. E. Crandall and J. P. Buhler, "Elementary function expansions for Madelung constants", J. Phys. A Math. Gen. 20:16 (1987), $5497-5510$.

[Crandall and Buhler 1994] R. E. Crandall and J. P. Buhler, "On the evaluation of Euler sums", Experiment. Math. 3:4 (1994), 275-285. 
[Crandall and Delord 1987] R. E. Crandall and J. F. Delord, "The potential within a crystal lattice", $J$. Phys. A Math. Gen. 20:9 (1987), 2279-2292.

[Forrester and Glasser 1982] P. J. Forrester and M. L. Glasser, "Some new lattice sums including an exact result for the electrostatic potential within the $\mathrm{NaCl}$ lattice", J. Phys. A Math. Gen. 15:3 (1982), 911-914.

[Glasser and Zucker 1980] M. Glasser and I. Zucker, "Lattice sums", Theor. Chem. Adv. Persp. 5 (1980), $67-139$.

[Kukhtin and Shramko 1993] V. V. Kukhtin and O. V. Shramko, "A new evaluation of the energy of Wigner BCC and FCC crystals", J. Phys. A Math. Gen. 26 (1993), L963-L965.
[Zucker 1976] I. J. Zucker, "Functional equations for poly-dimensional zeta functions and the evaluation of Madelung constants", J. Phys. A Math. Gen. 9:4 (1976), 499-505.

[Zucker 1984] I. J. Zucker, "Some infinite series of exponential and hyperbolic functions", SIAM J. Math. Anal. 15:2 (1984), 406-413.

[Zucker 1987] I. J. Zucker, "A systematic way of converting infinite series into infinite products", $J$. Phys. A Math. Gen. 20:1 (1987), L13-L17.

[Zucker 1990] I. J. Zucker, "Further relations amongst infinite series and products, II: The evaluation of three-dimensional lattice sums", J. Phys. A Math. Gen. 23:2 (1990), 117-132.

[Zucker 1998] I. J. Zucker, private communication, 1998.

Richard E. Crandall, Center for Advanced Computation, Reed College, Portland OR 97202, United States (Richard.Crandall@reed.edu)

Received September 4, 1998; accepted in revised form November 10, 1998 